\title{
Design and Simulation of Random Access Procedure in LTE
}

\author{
Mohamed Sami M. Yousef \\ Electronics Department, National \\ Telecommunication Institute (NTI); \\ Cairo, Egypt
}

\author{
Hussein A. Elsayed \\ Electronics and Communications \\ Engineering Department, Faculty \\ of Engineering, ain shams \\ University; Cairo, Egypt
}

\author{
Abdelhalim Zekry \\ Electronics and Communications \\ Engineering Department, Faculty \\ of Engineering, ain shams \\ University; Cairo, Egypt
}

\begin{abstract}
This paper introduces design and simulation of random access procedure taking place in Medium Access Control (MAC) sub-layer of Long Term Evaluation (LTE) User Equipment (UE) terminal. The random access procedure is required for completing connection establishment procedure occurring in the Radio Resource Control (RRC) layer to change from RRC_IDLE state to RRC_CONNECTED state. The design is based on 3GPP release 9 standards and implemented using Specification and Description Language (SDL). As an output from DL, the Message Sequence Chart (MSC) simulator trace shows that the built contention and non contention connection establishment, based on random access processes, prove their correct functionality and feasibility.
\end{abstract}

\section{Keywords}

LTE; the random access procedure; MAC Sub-layer; contention; non-contention; SDL

\section{ABBREVIATION TABLE}

\begin{tabular}{|l|l|}
\hline MAC & Medium Access Control \\
\hline LTE & Long Term Evolution \\
\hline UE & User Equipment \\
\hline RRC & Radio Resource Controlier \\
\hline SDL & Specification and Description Language \\
\hline MSC & Message Sequence Chart \\
\hline RLC & Radio link controller \\
\hline PDCP & Packet data compression protocol \\
\hline DRX & Discontinuous Reception \\
\hline WCDMA & Wideband code division multiple access \\
\hline 3GPP & 3rd Generation Partnership Project \\
\hline eNB & Evolved Node B \\
\hline PDU & Protocol Data Units \\
\hline BCH & Broadcast Channel \\
\hline DL-SCH & Downlink Shared Channel \\
\hline PCH & Paging Channel \\
\hline UL-SCH & Uplink Shared Channel \\
\hline RACH & Random Access Channel \\
\hline E- & Evolved UMTS Terrestrial Radio Access \\
\hline
\end{tabular}

\begin{tabular}{|l|l|}
\hline UTRAN & Network \\
\hline RNTI & Radio Network Temporary Identifier \\
\hline RA-RNTI & Random Access RNTI \\
\hline
\end{tabular}

\section{INTRODUCTION}

LTE was developed to support the increasing demand of packet data transmission besides the voice data. LTE is characterized by high-data-rate, low-latency and packetoptimized radio-access technology. This has its impact on LTE link-layer protocols design. Specifically, peak data rate of $100 \mathrm{Mb} / \mathrm{s}$ within $20 \mathrm{MHz}$ downlink spectrum allocation and an instantaneous uplink peak data rate of $50 \mathrm{Mb} / \mathrm{s}$ within a 20MHz uplink spectrum allocation [1].

The LTE network consists of three layers, namely, the radio resources controller (RRC), the data link-layer, Layer 2, and the physical layer, Layer1 as depicted in Figure 1. The data link layer itself comprises three sub-layers, namely, the MAC, the RLC and the PDCP. 3GPP defines two different MAC entities, one in the network side (eNB) and the other in the user side (UE). Here, the effort will be confined to the UE MAC sub-layer.

According to 3GPP specifications, UE MAC entity must perform certain procedures such as, but not limited to, Random Access procedure, Maintenance of Uplink Time Alignment, DL-SCH data transfer, UL-SCH data transfer, PCH reception, $\mathrm{BCH}$ reception, Discontinuous Reception (DRX) [2].

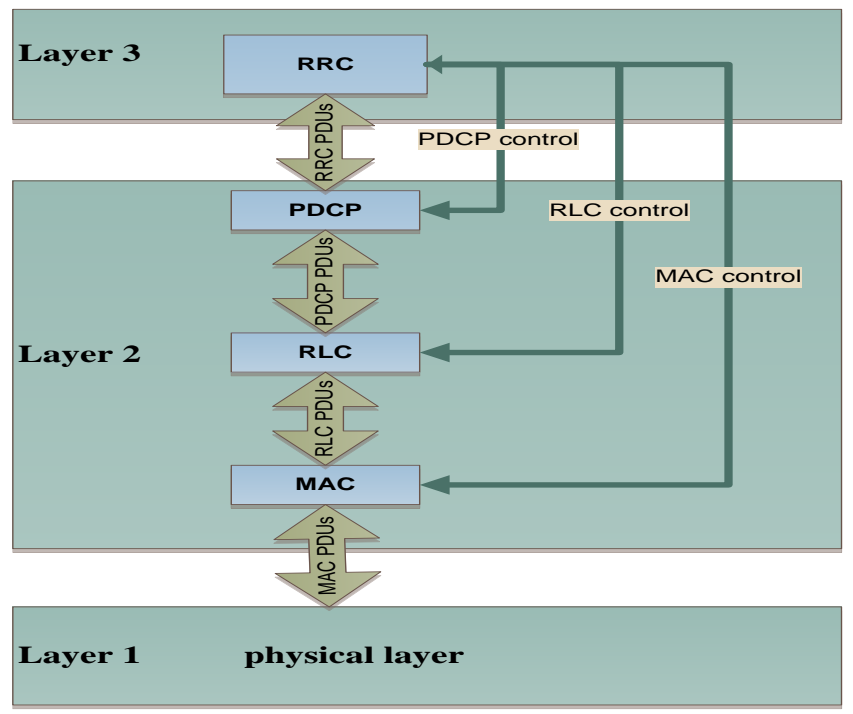

Figure 1 LTE layers 
The specification description high level language SDL has been used in communication systems design, implementation and testing as described in [3]. Specifically, SDL implementation of LTE control layers has been taking many researchers' attention. Design and Implementation of RLC sub-layer of WCDMA by using SDL is presented in [4].The LTE Mobile Terminals user plane has been modeled using SDL in [5], where layer 2, including its three sub-layers, and layer 3 for the mobile terminal have been developed. In [6], Random Access Procedure of Network side protocol is simulated to meet 3GPP Release 9. Random access procedure for LTE terminal using SDL design and implementation is introduced in [7], which focused mainly on the contention based random access procedure of 3GPP Release 8 .

Several researches proposed methods and architectures to improve both of contention and non contention based random access process. LTE clustering and non-clustering schemes performance of contention based random access procedure is evaluated in [8]. In [9], a survey is provided on Machine Type Communication (MTC) and focused on the 3GPP solutions proposed in order to fix the congestion problem caused by the massive number of MTC devices. An intensive comparison showing the strengths and weaknesses of proposed improvements for the operation of the random access channel of LTE and LTE-A is presented in [10]. [11] Shows how hierarchical control of different users will efficiently improve random access success probability and optimize system performance.

This paper focuses on 3GPP Release 9 UE MAC sub-layer Random Access procedure implementation. Random access procedures in the contention and non contention based mode will be carried out using specification and description language (SDL).

The paper is organized as follows: Section 3 provides a survey on the recent random access process in LTE. The LTE UE side entity structure is shown in section 4. Section 5 introduces the UE MAC structure recommended by $3 \mathrm{GPP}$ and the implemented one. The SDL states of the implemented random access processes are explained in section 6 , while section 7 discusses the simulation results of the two random access processes .Finally, the conclusions and future work is presented in Section 8.

\section{LTE RANDOM ACCESS}

In this section the random access process will be introduced. The UE needs to be synchronized in the uplink direction for transferring any application data. This requires UE transition from RRC_IDLE to RRC_CONNECTED mode [12]. Accordingly, the UE sends RRC connection request message during the random access procedure performed in the MAC sublayer.[13]

Depending on whether the phrases ra-PreambleIndex or raPRACHMaskIndex were indicated in the random access request or not the process will be non contention based or contention based, respectively.

\subsection{Contention-based Random Access Procedure}

In this process, there is no reserved ra-PreambleIndex and raPRACHMaskIndex for the UE. It randomly selects a RA preamble and PRACH resource from a pool defined by the eNB. The process consists of 4 steps to send the request and resolve the contention including their acknowledgments as seen in Figure 2.
Contention resolution is needed as multiple UEs in the cell could select the same ra-PreambleIndex and raPRACHMaskIndex.

\subsection{Non Contention-based Random Access Procedure}

In this process UE has already assigned ra-PreambleIndex and ra-PRACHMaskIndex from the network, so the process will complete in only three steps as can be seen in Figure 3.

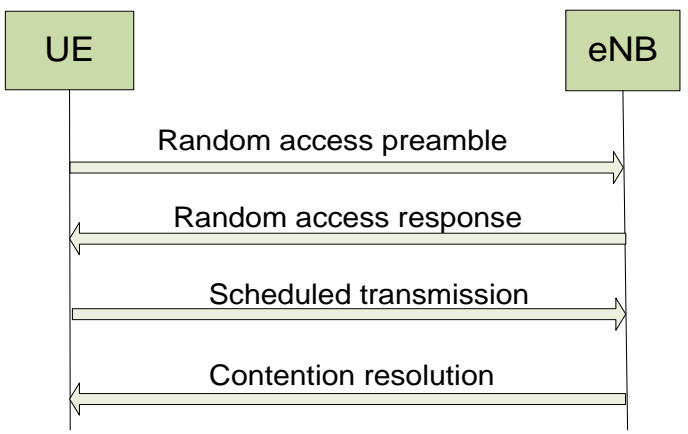

Figure 2 Contention-based Random Access Procedure steps

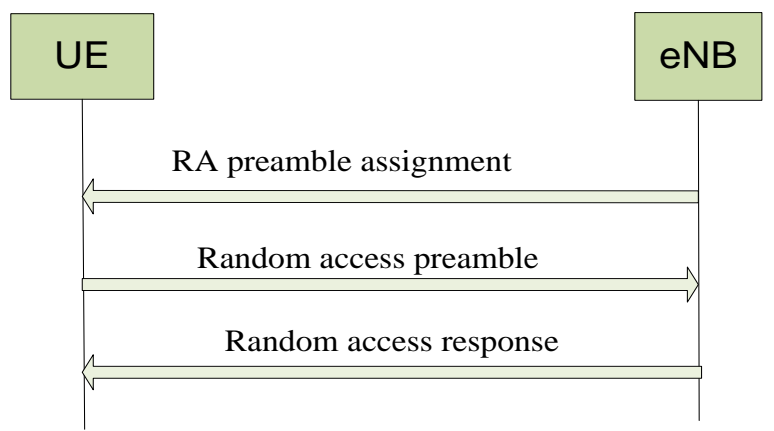

Figure 3 non Contention-based Random Access Procedure steps

\section{UE LTE SYSTEM MAC MODEL}

The implemented UE LTE system mac is shown in

Figure 4. This system consists of RRC, MAC, and physical block. These are the blocks sharing in random access process.

In the control plane, the RRC block configures the MAC block by signaling messages and carries all the configuration parameters of the layer 3 and the layer 1 .

Some of the primitives used between MAC and RRC are listed below:

CMAC_RANDOM_ACC_REQ is a request from RRC to MAC in order to start the contention based random access. CMAC_RANDOM_ACC_REQ_non_cont is an indication from RRC to MAC in order to start the non contention based random access. Successful_Random_Access_Process: the MAC sends an indication to RRC that the process has been completed successfully.

Non_Successful_Random_Access_Process: the MAC sends an indication to RRC that the process has not been completed successfully.

Other Primitives between MAC and physical block are: preamble_value: MAC instructs the physical block with the preamble value. 
Random_Access_Response_MAC_PDU: The physical block indicates the reception of the random access response to the MAC. MAC_PDU_DL: the physical block indicates the reception of normal PDU to the MAC. MAC_PDU_UL: the MAC instructs the physical block with a new PDU to send.

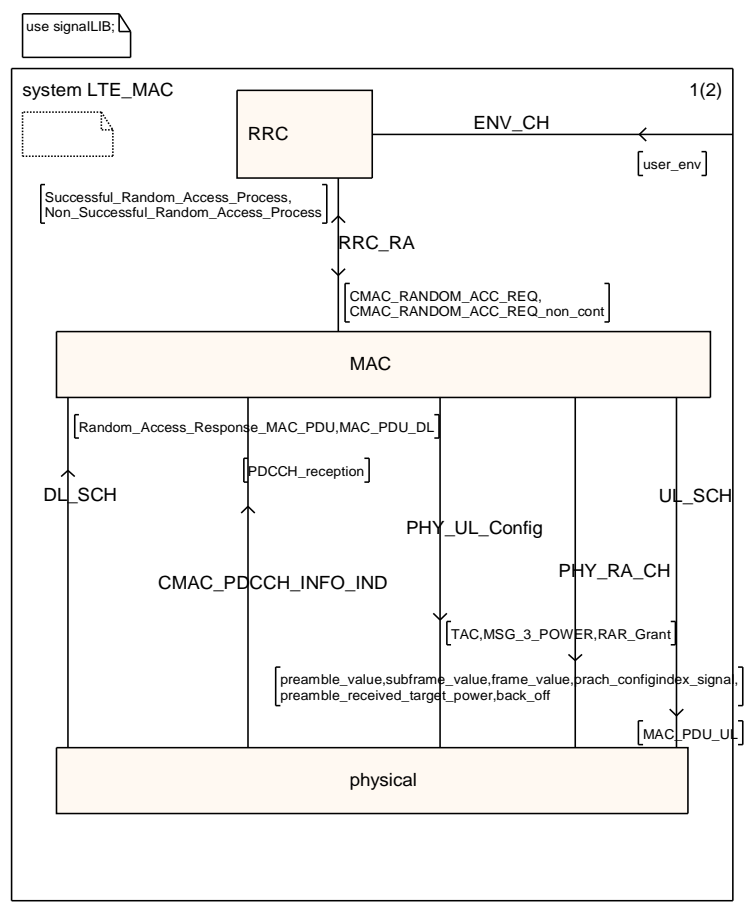

Figure 4 the implemented UE system

\section{UE MAC BLOCK DESIGN}

E-UTRAN defines two MAC entities; one is in the UE and the other in the E-UTRAN side. The functions performed by each of those entities are different from each others.

Figure 5 shows that the UE side mac functions proposed by $3 \mathrm{GPP}$, are but not limited to, Mapping between logical channels and transport channels, Multiplexing, Demultiplexing, Error correction through HARQ, Logical Channel prioritization and Scheduling information reporting [2].

In this paper the random access process is designed and simulated as it can be considered one of the major processes in the MAC block. Figure 6 shows the implemented random access process along with primitives to each of the multiplexing/ demulteplixing process, physical block, and RRC block.

\section{RANDOM ACCESS PROCESS STATES IMPLEMENTATION}

Since SDL is based on state diagrams, Figure $7 \&$

Figure 8 are proposed to fully implement normal Non contention based random access process and contention based process respectively.

According to the Figure, the states are:

Idle: MAC layer in starting state, Random_Access_Response_Reception: the MAC layer has received the random access request (either contention or non contention), sent the Random access preamble and waiting the random access response. For the non contention based random access procedure, successful random access response is a successful termination of the random access procedure and returning to Idle state, Wait_msg3_Reply: after receiving the random access response, "in case of contention based process" Random_access_control process will send request to the Multiplexing_and_assembly process to send msg3 and to take a copy to store in msg3 buffer,msg4_Waiting: msg3 has been sent and the MAC layer is waiting contention resolution message (msg4). In case of successful contention resolution the random access procedure is also successfully finished and returns to Idle state.

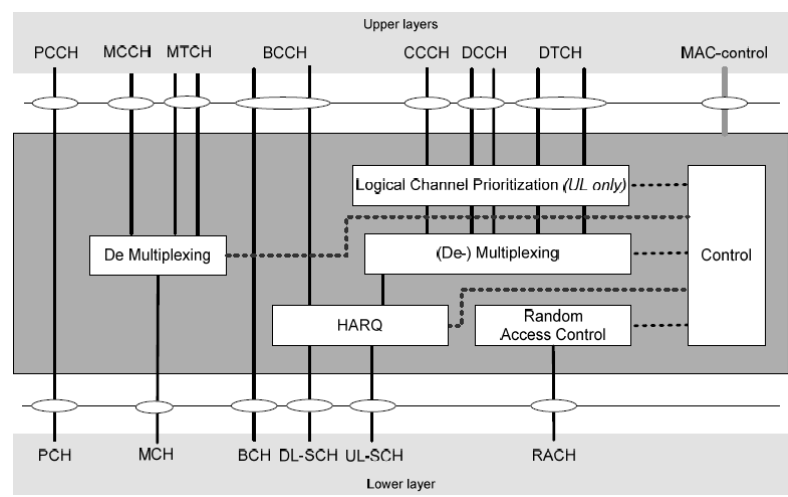

Figure 5. 3GPP UE side MAC entity

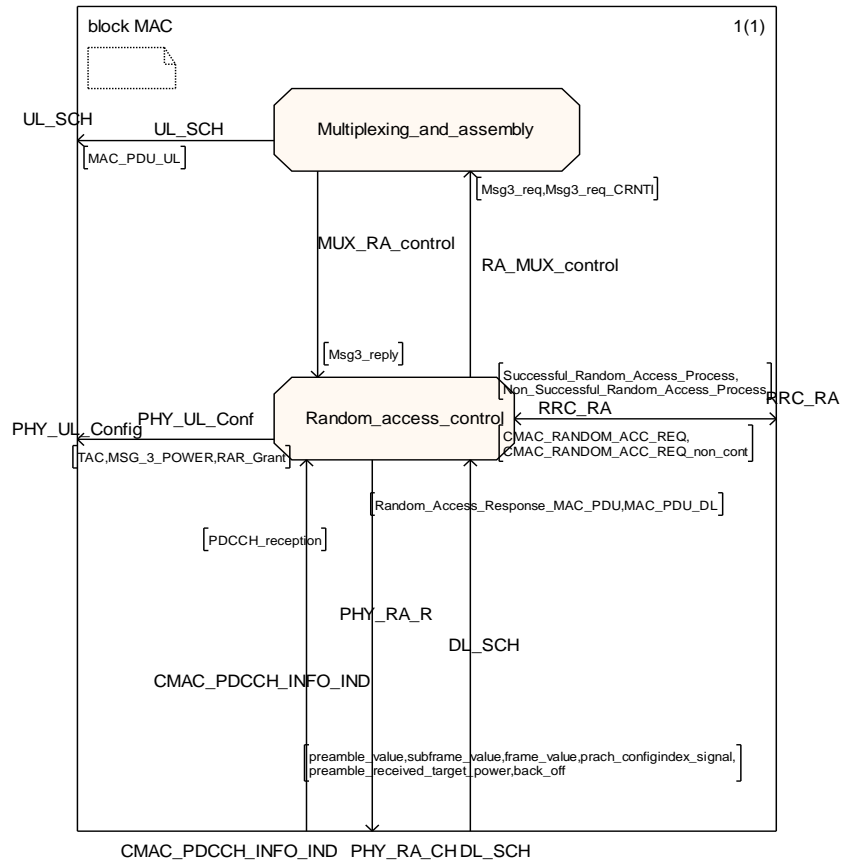

Figure 6 implemented MAC block

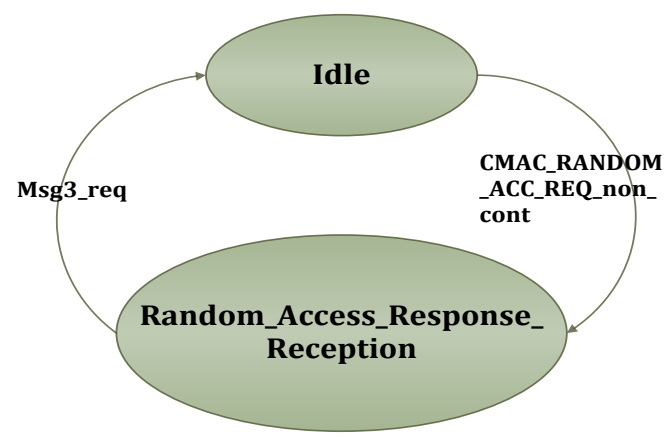

Figure 7 proposed non contention based random access process states 


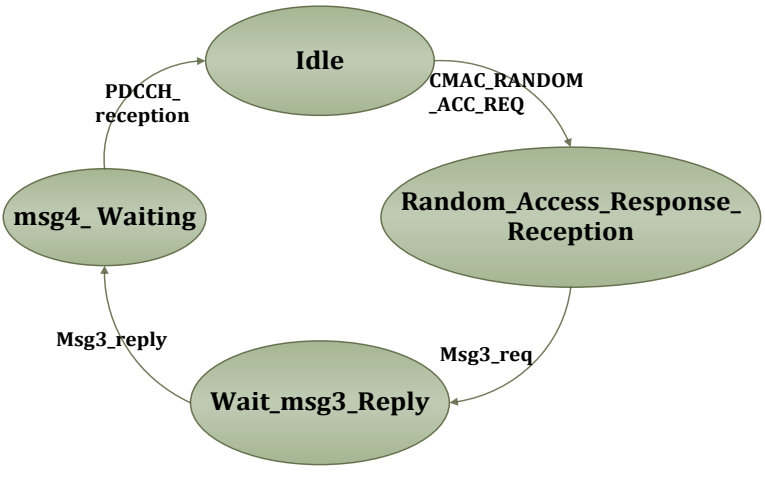

Figure 8 proposed contention based random access process states

Figure 9 shows the random selection of the Preamble index (ra_preambleindex) for the contention based random access process, where SDL operator "randint" is used to generate random integer number to be the ra-PreambleIndex from the network specified range from preamble_start to preamble_end. The input of prach_config_index procedure is prach_configindex while its outputs are available_frame_no and available_subframe_no, which are used to determine the available frames and sub frames for the random access preamble transmission [14].

\section{SIMULATION RESULTS}

SDL provides functional simulation, which uses Message Sequence Chart (MSC) simulator trace introduced by Telelogic Tau SDL and TTCN Suite 4.0 which is launched by the Telelogic Tau Company.

Figure 10 shows the simulation results for successful non contention based random access process. After the MAC layer receives the request (CMAC_RANDOM_ACC_REQ_non_cont) for non contention based random access procedure signal from RRC with the prescribed ra-PreambleIndex value (101010), the MAC sends preamble_value together with its parameters comprising subframe_value, frame_value and preamble_received_target_power to the physical layer. Then it starts Random Access Response window timer (RAR_window_timer). Now MAC changes from Idle state to Random_Access_Response_reception state, where it is waiting for the random access response PDU (Random_Access_Response_MAC_PDU) including the pre transmitted preamble. If the Random Access Response reception is considered successful, RAR_window_timer is stopped and Successful_Random_Access_Process signal is sent to RRC as an indication of successful completion of Random Access procedure.

Figure 11 shows successful contention based random access process. CMAC_RANDOM_ACC_REQ signal is the request for the process from RRC to MAC. It is the MAC role to randomly select ra-PreambleIndex. As for non contention based, the MAC sends preamble_value (011100) and its parameters to the physical layer. While after successful Random Access Response reception, Contention Resolution step is still needed, this leads Random access control process to send Msg3_req signal to Multiplexing and assembly process as a request for transmitting the PDU. While it is now in Wait_msg3_Reply state. The Multiplexing and assembly block will send MAC_PDU_UL signal (Msg3) to PHY process to transmit the PDU and reply to the Random access control process with Msg3_reply signal which includes the transmitted PDU that will be buffered for the contention resolution step. The timer mac_ContentionResolutionTimer now starts waiting for MAC_PDU_DL message while Random access control process is now in msg4_Waiting state.

Based on 3GPP standard, the contention resolution step is considered to be successful if the contention resolution identity field in the received PDU matches the one which was sent earlier in the MSg3, consequently, Successful_Random_Access_Process signal is transmitted to RRC process [2].

\section{CONCLUSION AND FUTURE WORK}

In this paper, SDL and MSC are used to design and implement the random access process, which is one of the important MAC sub-layer procedures in LTE system. Optimized model is achieved by integrating both Contention and non-contention based random access processes to avoid recurrence of joint operations in order to improve the performance. SDL/MSC is used to verify and validate the functionality of the proposed scheme in different conditions including normal successful flow and abnormal cases.

The introduced methodology can be used to implement other processes in the MAC sub-layer or any control layer protocols of LTE system. The follow up of this work should be extended to the rest of the functions of the MAC layer in conjunction with the other layers of LTE UE and implemented on a real system, then tested to evaluate the system performance. A further step can be taken as feedback to the design phase to improve the system performance.

\section{REFRENCES}

[1] 3GPP. TR 25913 V9.0.0 3rd Generation Partnership Project; Technical Specification Group Radio Access Network; Requirements for Evolved UTRA (E-UTRA) and Evolved UTRAN (E-UTRAN); (2010-02)

[2] 3GPP. TS 36.321 V 9.6.0 3rd Generation Partnership Project; Technical Specification Group Radio Access Network; Evolved Universal Terrestrial Radio Access (E-UTRA);Medium Access Control (MAC) protocol specification; (2012-03)

[3] ETSI EG 201 383: "Methods for Testing and Specification (MTS); Use of SDL in ETSI deliverables; Guidelines for facilitating validation and the development of conformance tests".

[4] Amin S. Ibrahim, Abdelhalim Zekry, Hussein A. Elsayed, "Design and Implementation of Radio Link Control as a Part of the WCDMA Radio Interface Protocols by using SDL," International Journal of Computer Applications., Volume 56- No.16, October 2012

[5] Anas Showk, David Szczesny, Shadi Traboulsi, Irv Badr,Elizabeth Gonzalez, and Attila Bilgic "Modeling LTE Protocol for Mobile Terminals Using a Formal Description Technique". In SDL 2009: Design for Motes and Mobiles (p. 255). Germany: Springer Berlin Heidelberg.

[6] Fa-Tang Chen, Zheng Zhang. "Design and Simulation of Random Access Procedure in TD-LTE". Computational and Information Sciences (ICCIS), 2012 Fourth International Conference on (p. 4). Chongqing : IEEE

[7] Wu WEN, B. Dan-dan NIU. "Schematic design and implementation of random access in LTE terminal protocol stack". Mobile Congress (GMC), 2010 Global 
(p. 4). Shanghai: ieee.

[8] Ahsan Nawaz Khan, Junaid Khalid, Hassaan Khaliq Qureshi "Performance Analysis of Contention-Based Random Access Procedure in Clustered LTE Networks" Seventh International Conference on Next Generation Mobile Apps, Services and Technologies,2013

[9] "Towards a better support of Machine Type Communication in LTE-Networks: Analysis of Random Access Mechanisms" 2nd international conference on advances in Biomedical Engineering,2013

[10] "Is the Random Access Channel of LTE and LTE-A Suitable for M2M Communications? A Survey of Alternatives" IEEE COMMUNICATIONS SURVEYS \& TUTORIALS, VOL. 16, NO. 1, FIRST QUARTER
2014

[11] Zhide Chen and Yali Zeng "Random Access Control for M2M in LTE System" International Journal of Distributed Sensor Networks, 2013

[12] 3GPP TS 36.331: "Evolved Universal Terrestrial Radio Access (E-UTRA); Radio Resource Control (RRC); Protocol specification".

[13] SeungJune Yi ,SungDuck Chun, YoungDae Lee, SungJun Park, SungHoon Jung, (2012). Radio protocols for LTE and LTE-Advanced.

[14] Zhongda Du, Shenzhen (CN); Rui Ma, ShenZhen (CN); Bin $\mathrm{Yu}$, ShenZhen (CN), "METHOD AND TERMINAL FOR SELECTING RANDOM ACCESS RESOURCE," U.S. Patent 8,570,902 B2, Oct. 29, 2013.

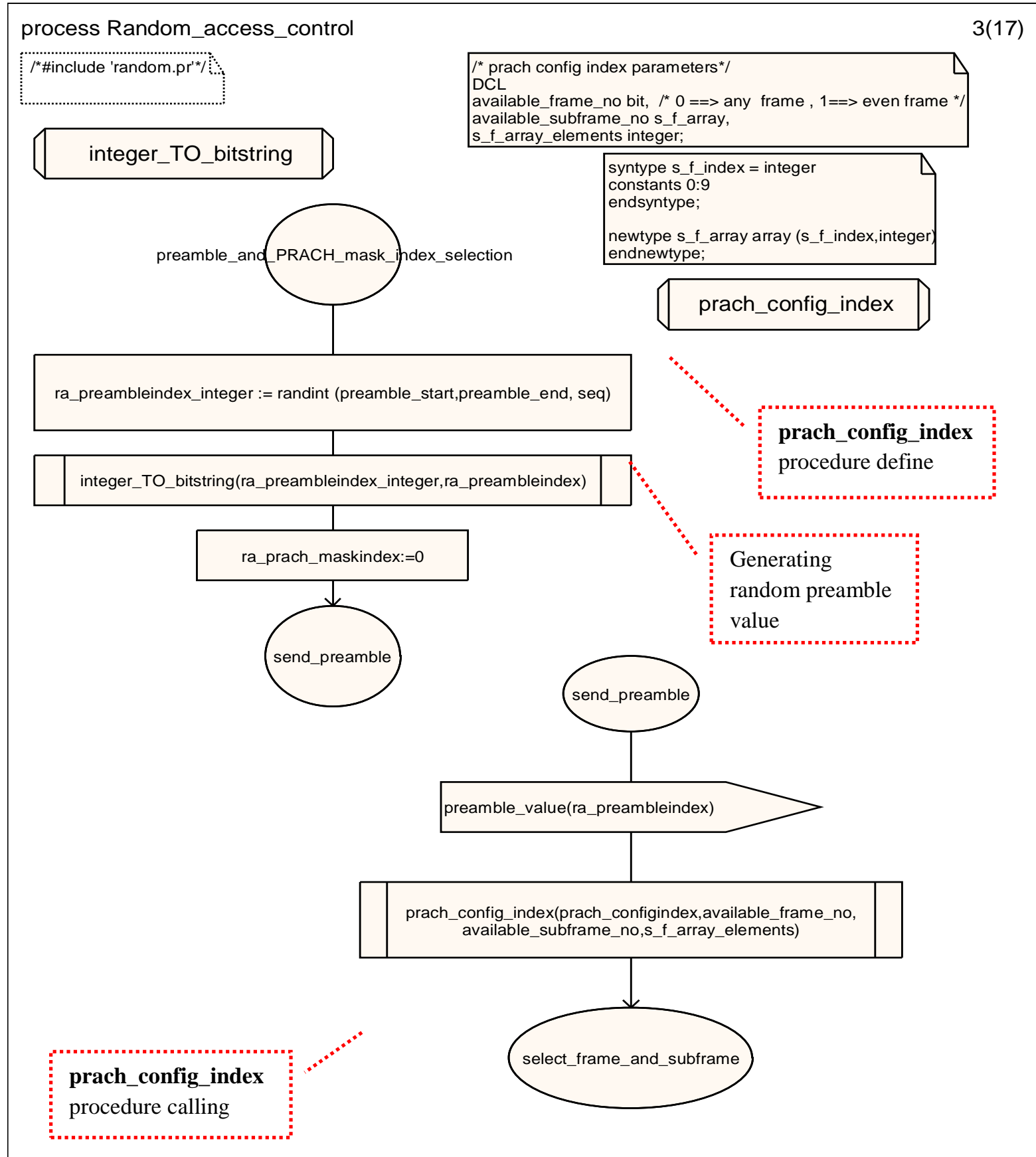

Figure 9 Proposed SDL flow chart of the random selection of preamble index and the selection of the available sub-frame 


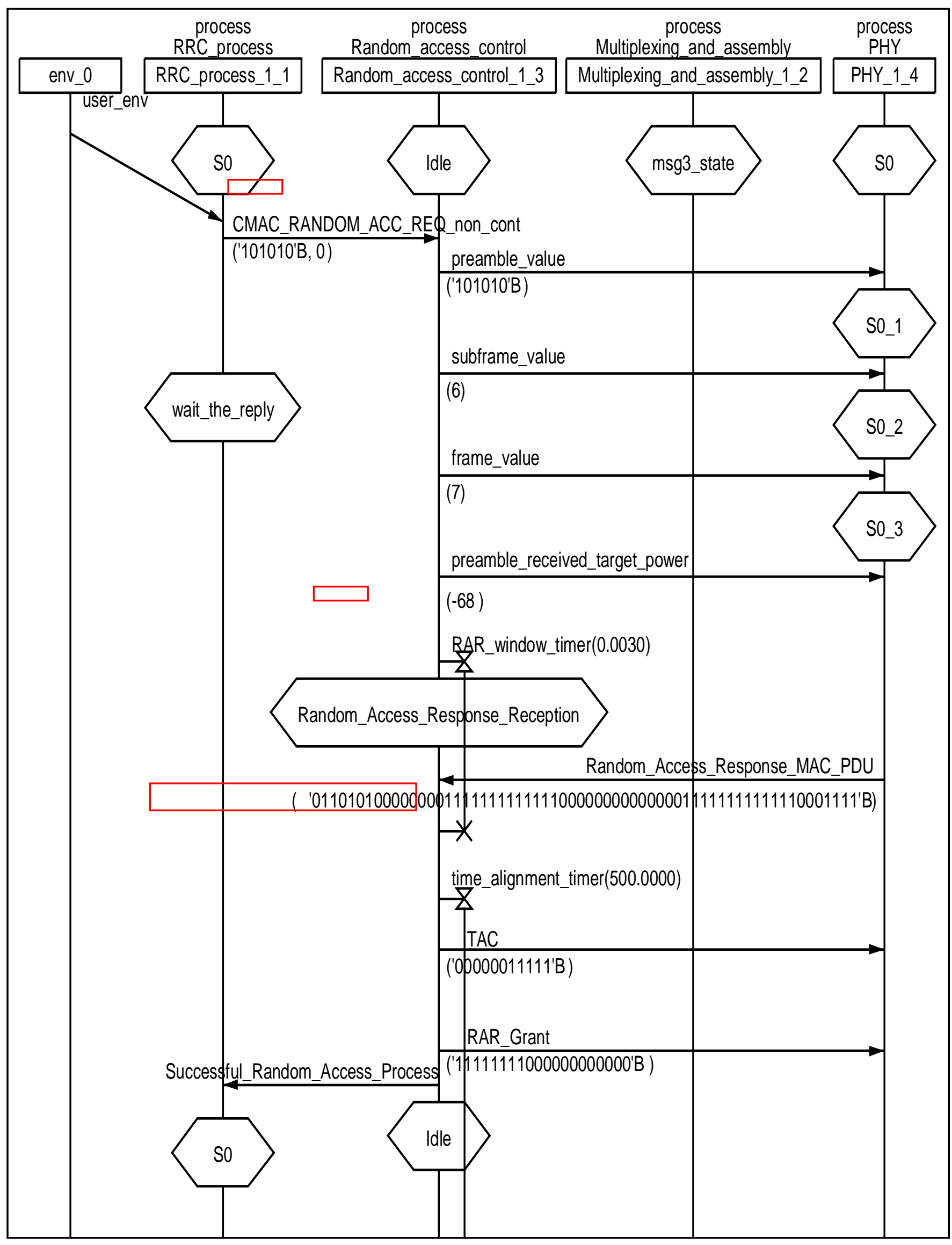

Figure 10 non contention based random access process simulation results 


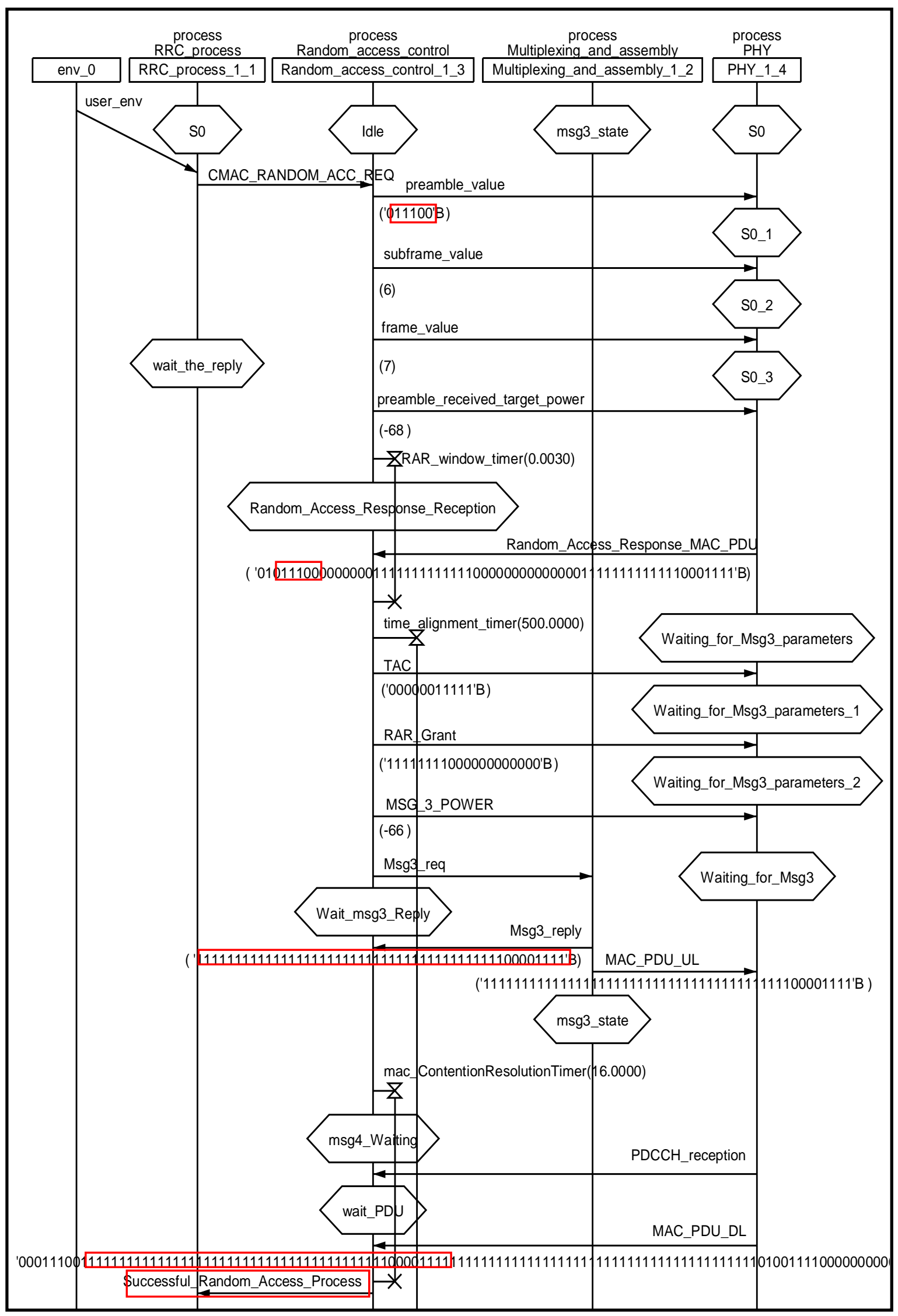

Figure 11 contention based random access process simulation results 